\title{
Téoros
}

Revue de recherche en tourisme

\section{Le nouveau Pôle Maisonneuve deviendra la masse critique touristique la plus importante de Montréal}

\section{Guy R. Morin}

Volume 10, numéro 3, novembre 1991

Destination Montréal : d'hier à demain

URI : https://id.erudit.org/iderudit/1079204ar

DOI : https://doi.org/10.7202/1079204ar

Aller au sommaire du numéro

Éditeur(s)

Université du Québec à Montréal

ISSN

0712-8657 (imprimé)

1923-2705 (numérique)

Découvrir la revue

Citer cet article

Morin, G. R. (1991). Le nouveau Pôle Maisonneuve deviendra la masse critique touristique la plus importante de Montréal. Téoros, 10(3), 47-50.

https://doi.org/10.7202/1079204ar d'utilisation que vous pouvez consulter en ligne. 


\section{Le nouveau Pôle Maisonneuve deviendra la masse critique touristique la plus importante de Montréal}

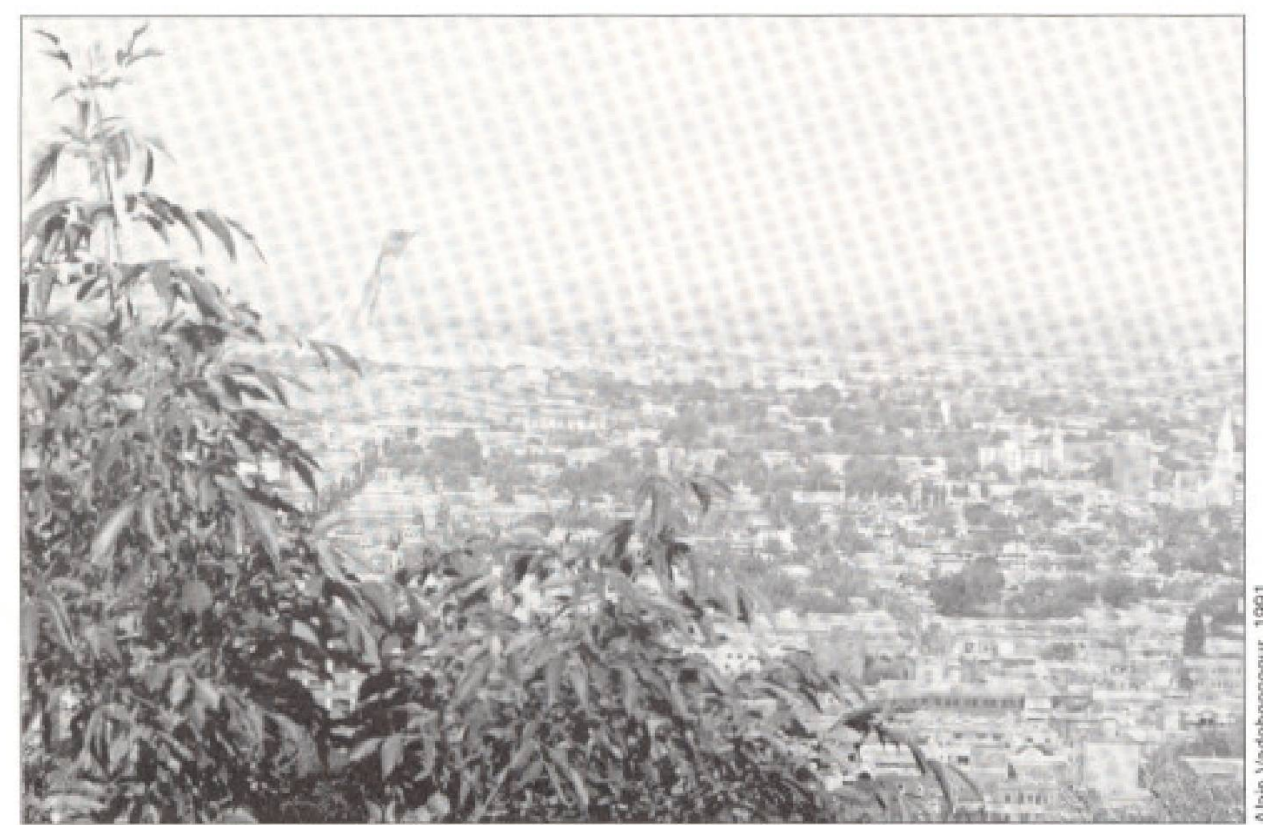

Du polle naturel Mont-Rogal, whe silhouelle fascinante...

\section{Le dernier-né des pôles touristiques du Grand Montréal}

Le 26 juin 1991, le Comité exécutif de la Ville de Montréal approuvait le nom Pôle Maisonneuve pour désigner le grand quadrilatère compris entre le boulevard Pie$\mathrm{IX}$, le boulevard Rosemont, la rue Viau et l'avenue Pierre-de-Coubertin.

Dès lors le ministère du Tourisme et le ministère des Transports du Québec ont entrepris l'implantation d'un nouveau programme de signalisation routière des trois principaux pôles touristiques de Montréalle Centre-ville, le Vieux-Montréal et le Pôle Maisonneuve-auquel s'ajoutera la signalisation piétonnière de la Ville qui inclura deux autres pôles: Mont-Royal / Oratoire et Parc-des-Iles. Ces deux programmes qui devraient être en place avant l'été 1992 optimiseront encore l'achalandage du Pôle Maisonneuve qui recevra 4,6 millions de visiteurs payants cette année: 3,3 millions au Parc olympique

Monsieur Guy A. Morin est adjoint au président. Planification et développement, ấla Regie des Installations Olympiques. et 1,3 million au Jardin botanique dont un demi-million à l'Insectarium, pour la deuxième année consécutive.

L'ouverture du Biodôme en 1992 ajoutera 1,1 million de visiteurs à l'achalandage actuel pourun total de 5,7 millions d'entrées par année. D'autres projets à l'étude pourraient ajouter un autre million de visiteurs à ces chiffres.

\section{Le magnétisme du Pôle Maisonneuve}

Le jour comme la nuilt, à des kilometres à la ronde, une silhouette fascinante se profile dans le ciel montréalais. C'est la plus haute tour inclinée au monde. Comme un immense clocher, elle guide vers le Pôle Maisonneuve, un site au véritable magnétisme qui regroupe le deuxième jardin botanique en importance au monde, un oasis en plein centre urbain et un parc olympique coté deux fois trois étoiles.

Le Pôle Maisonneuve, c'est 188 hectares de loisirs pour les Montréalais et l'une des plus importantes destinations touristiques du Québec. Le Jardin botanique occupe 73

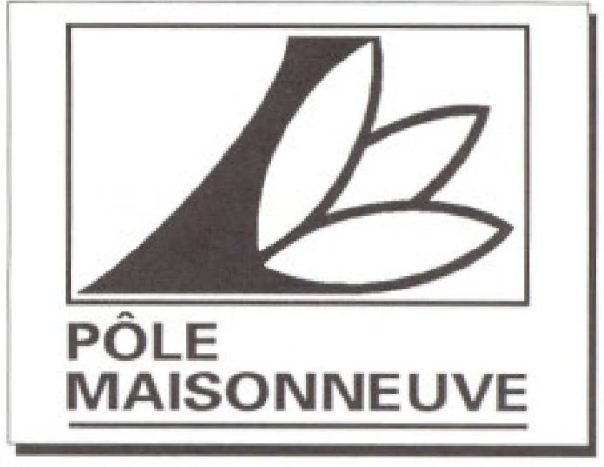

hectares, le Parc Maisonneuve 60 hectares et le Parc olympique 55 hectares.

\section{Un nom qui en dit long}

A l'occasion du 350 e anniversaire de Montréal, le Pôle Maisonneuve s'ajoute donc aux autres noms touristiques comme Centre-ville, Vieux-Montréal,Parc-des-Iles et Mont-Royal/Oratoire.

La Tour du Stade olympique symbolise le Pôle et une fleur en Éclosion traduit le dynamisme des équipements dédiés à la vulgarisation des sciences dans un cadre ecologique.

Outre ladésignation d'une véritable Citédu sport, du loisir et de l'environnement, le nom Pôle Maisonneuve rappelle au visiteur l'oeuvre du fondateur de Montréal, Paul de Chomedey, sieur de Maisonneuve. Il ť́moigne aussi de l'histoire récentedu quartier qui, jusqu"en 1912, faisait partie de la Ville de Maisonneuve. Le groupement dans un même site d'autant d'installations de haut calibre et de vocations différentes représente un atout touristique majeur. L'appellation «pôle» traduit cette volonté.

- L'appellation «pôles met l'emphase sur le secteur toutentier plutôt que sur la thématique ou la réputation d'un équipement specifique.

- Le mot apôlen a de multiples sens, comme ceux de pôle d'attraction et de pôle de développement. Plusieurs se rapportentàdes contenus scientifiques, notamment en astronomie et en phy- 
sique. Cette diversité d'interprétations $\mathrm{s}^{\dagger}$ accorde bien aे celle des points d'intérêt du Pôle Maisonneuve.

\section{Unique au monde, uniquement à Montréal!}

L'unicité et l'authenticité sont les traits dominants du Pôle Maisonneuve:

- l'architecture futuriste du Stade, de sa Tour et du Biodôme voisine l'architecture traditionnelle du Jardin de Chine et du Pavillon japonais;

- la recherche scientifique soustend la démarche didactique du Jardin botanique, de l'Insectarium et du Biodôme;

- le Pôle est le secteur montréalais qui reçoit déjà le plus grand nombre de visiteurs payants annuellement et ce

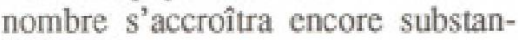
tiellement.

\section{Le Jardin botanique comprend...}

Les serres, la flore du monde entier et les joyaux de la plus importante collection de bonsaïs en Occident!

Les jardins extérieurs, la roseraie, le ruisseau fleuri, le jardin alpin à parcourir à pied ou en ski de randonnée!

Le Jardin japonais, la nature dans sa beauté la plus pure et la cérémonie du thé dans un pavillon authentique!

Le Jardin de Chine, dans la tradition des jardins du sud du Yangtze, sept pavillons pour découvrir une culture millénaire!

L'Insectarium, une des plus importantes collections d'insectes, des specimens rares aux plus spectaculaires!

\section{Le Parc olympique, inclut...}

Le Stade, une architecture unique au monde et le plus grand hall au Canada sous un meme toit... ouvrant!

La Tour olympique, la plus haute tour inclinée au monde, neuf fois plus penchée que la célebre tour de Pise!

Le Centre de natation, un centre de conditionnement physique et quatre piscines intérieures pour le plaisir et la competition à l'occasion!
Le Biodôme, une incursion à couper le souffle dans la vie animale et végétale des quatre climats de notre planète bleue!

\section{Le Parc Maisonneuve, c'est...}

Un clin d'oeil au béton et aux feux de circulation! Cyclistes et piétons sillonnent ses pistes et ses allées spécialement aménagées, en toute tranquillité. C'est un poumon de verdure pour toute la famille au coeur de l'une des trois villes qui ont la meilleure qualite de vie au monde!

Voilà donc la présentation sommaire du Pôle Maisonneuve d'aujourd'hui qui s'enrichira sans doute encore de nouvelles attractions importantes!

\section{La clientèle du Pôle Maisonneuve}

Nous faisons une différence fondamentale entre les événements et les activités. Les premiers sont ponctuels tandis que les seconds sont offerts à l'année longue. Les événements ponctuels attirent 75 pour cent de l'achalandage de 3,3 millions du Parc tandis que les activités permanentes constituent 90 pour cent de $l^{2}$ achalandage de 1,3 million du Jardin. AuParc, $c^{\dagger}$ est la visite de l'observatoire de la Tour et la natation qui constituent les principales activités mais l'ouverture du Biodôme contribuera toutefois à ramener cette proportion autour de 45 pour cent des 4,4 millions de visiteurs escomptés annuellement. Le Stade canalise la quasi-totalité de I'achalandage aux événements du Parc tandis que ce sont les expositions florales periodiques dans les serres qui constituent les événements du Jardin.

Le spectateur reste quatre heures à une activité comme le baseball, un grand salon ou un concert rock. La visite des serres, du Jardin de Chine, du Jardin japonais et de l'Insectarium constitue les principales activités du Jardin qui peuvent totaliser une journée entière. Le séjour à l'observatoire et au Centre d'interprétation du Parc, au sommet de la Tour, dure 45 minutes en moyenne et la visite guidée prend une heure. La baignade dure trois heures en moyenne et on prévoit que la visite complète du Biodôme prendra entre deux et trois heures au moins.

On constate donc que ale temps de tout voir et tout faires au Pôle Maisonneuve prendra facilement deux jours dès l'an prochain. Cette réalité pourrait justifier l'implantation de facilités d'hébergement sur le site même. Ceséventuelles facilités devraient toutefois être de type familial, compte tenu que c'est principalement en famille qu'on vient au Pôle Maisonneuve à longueur de semaines pendant la saison haute et pendant les fins de semaine de l'automne au printemps. Les groupes scolaires prennent la relèvependant la semaine durant la saison académique et nous souhaitons y ajouter la clientèle des aînés en privilégiant des facilités d'accueil atout à l'intérieurs dans nos projets de développement futur.

L'écart entre la saison haute et la saison basse est actuellement plus contrasté au Parc olympique qu'au Jardin botanique. En effet, les deux-tièrs de l'achalandage annuel de la Tour est présentement concentré dans le tiers de l'année tandis qu"au Jardin, c'est la moitié de l'achalandage annuel qui est concentré dans les cinq meilleurs mois.

\section{Voulez-vous partager ma vision?}

Ma vision du Pôle Maisonneuve de demain repose sur les quatre défis que j'ai proposés au Parc olympique il y a déjà cinq ans:

- insuffler une âme au béton,

- inventer notre avenir,

- renforcer le Montréal touristique,

- consolider la vision d'ensemble.

\section{Notre premier défi: insuffler une âme au béton}

L'âme d'un édifice procède plus de sa vocation que de l'addition des activités qui s"y déroulent. La première étincelle de vie du Parc olympique fut, bien entendu, les Jeux olympiques de 1976 ! Cette étincelle fit un tel éclat qu'il fut impossible d'estomper l'ombre qui suivit.

Le coût des Jeux et les enquêtes qu'ils entraînèrent, des temps économiques difficiles et des appréhensions technologiques nombreuses laissèrent le Stade sans toit pendant onze ans, le condamnant de facto à ne servir que pendant la moitié de l'année. Le sport professionnel fut done l'âme du Stade olympique pendant tout ce temps avec le SuperMotocross annuel et quelques concerts de passage au fil des étés. Il y avait bien juste à côté, aux piscines et dans le Vélodrome, les athlètes de la natation et du cyclisme de compétition, mais leur petit nombre laissa graduellement la place aux bains libres et aux salons commerciaux. 
Il y eut bien une expérience d'animation hivernale dans le vaste Stade décoiffé où l'on aménagea une vaste patinoire populaire pendant quelques années, mais les coûts d'eclairage étaient sans commune mesure avec les revenus de cette activité, On dût done l'abandonner.

L'été, il y avait occasionnellement de grandes foules, surtout au début des années ' 80 , lorsque le football et le soccer ont ajouté leurs achalandages respectif à à celui des Expos qui atteignit 2,3 millions d'admissions en 1983 mais le béton reprenait tous ses droits la plus grande partie du temps. Pendant tout ce temps-là et jusqu'en 1986, le Jardin botanique et ses serres étalaient verdure et couleurs sans chercherà dialoguer avec le voisin qui ne se forçait pas davantage...

Ces deux solitudes, la verte et la grise, se sont donc voisinées sans se parler pendant dix ans, sauf une fois en 1980, pour tenir les Floralies internationales au Vélodrome où plus de cent mille visiteurs en une semaine vinrent au Parc olympique pour des fleurs!

C'est au cours de l'automne 1986 que la Régie des installations olympiques balbutia quelques signaux à son voisin au sujetdu Vélodrome... Et le Jardin botanique parla de ses projets de Jardins japonais et chinois, d'Insectarium, etc. Telles furent les premières parcelles de réalisations de notre premier objectif.

L'installation d'un toit sur le Stade et le parachèvement de la Tour insufflèrent une nouvelle vie qui s'épanouit rapidement à partir de 1989:

- reconnaissance comme attraction trois étoiles par le Guide Michelin;

- invitation à joindre les rangs des fondateurs de la Fédération des Grandes Tours du Monde;

- arrivée des cinq plus grands salons publics de Montreal dans le Stade qui $\mathrm{y}$ attirent trois quarts de million de visiteurs en hiver;

- record d'occupation des stades en Amérique du Nord en 1990;

- accueil du millionième visiteur à l'observatoire de la Tour, deux ans après son ouverture;

- parachèvement de l'arrière du Stade pour de nouveaux développements;

- ouverture prochaine du Biodôme et nouveaux achalandages importants.
Malgré les défaillances de la technique et du scepticisme qu'elles alimentent, nous vivons des moments excitants au Parc olympique et chez notre voisin, le Jardin botanique de Montréal, parce que nous sommes devenus des attraits touristiques de classe internationale.

\section{Notre second défi: inventer notre avenir}

Alan Kay, directeur de la recherche chez Apple Computer, a déjà dit: «le meilleur moyen de prédire l'avenir, c'est de l'inventer". Au Parc olympique, nous devons «réinventer» notre avenir pour arriver où nous voulons être demain, compte tenu de l'effort de récupération requis.

En effet, les difficultés techniques propres à un équipement de la taille et de la complexité des équipements dont a hérité la Régie des installations olympiques, equipements sans précédent au monde, feront toujours les gorges chaudes des médias... La Tour Eiffel a suscité les mêmes doutes pendant un demi-siècle!

Outre la détermination, il nous faut donc une bonne dose d'humour et le publicitaire français Jacques Séguéla nous encourage sans le savoir avec sa «théorie des trois Rs».qui postule que les grands monuments du monde s'ils veulent atteindre la notoriété, doivent: comporter un risque, matérialiser un rêve et faire rire... Dans ces conditions, on ne peut douter de notre notoriété!

Sur un ton plus sérieux, nous croyons que le temps était venu en 1986, de mettre à jour la vision olympique et d'oublier un certain passé pour rendre le Parc plus vivant, plus attirant, moins déficitaire.

C'est alors que fut créée une vếritable direction du marketing au Parc olympique avec mission de développer, de positionner, d'implanter et de révaluer.

A l'instigation de son président, M. Pierre Bibeau, la nouvelle mission de la Régie s'appuie sur ces acquis pour vouloir aller plus loin etc'est dans ce but que la réorganisation administrative du ler novembre 1991 a créé le poste d'adjoint au président, pour la planification et le développement.

Compte tenu de l'énoncé de mission de la Régie, les projets de développement doivent répondre à trois paramètres:
1. constituer une attraction rentable pour la Régiee tgénératrice d'achalandages complémentaires aux vocations actuelles et potentielles du Pôle Maisonneuve;

2. mettre en valeur de manière permanente les sites du Parc olympique en tenant compte des conditions logistiques et financières des opérations actuelles et potentielles:

3. faciliter des ententes de partenariat à long terme avec le secteur privé en matières d'immobilisation et de gestion,

\section{Notre troisième défi: renforcer le Montréal touristique}

Depuis 1986, plusieurs gestes démontrent que ce défi est bien relevé:

- l'aménagement du Hall touristique et la reconnaissance du Guide Michelin ont accrédité la force d'attraction du Parc et de sa Tour;

- le développement du Jardin botanique de Montréal s'est inscrit en parfaite harmonie avec le volet tourisme et loisirs du Parc olympique;

- la Régie est un partenaire de premier niveau de l'Office des Congrès et du Tourisme du Grand Montréal en participant à ses orientations et à ses efforts promotionnels;

- la Régie relève du Ministère du Tourisme depuis l'automne 1989 et la publicité du Québec sur les marchés extérieurs met bien la plus haute tour inclinée au monde en valeur;

- l'intégration du Jardin et du Parc sous le vocable Pôle Maisonneuveen 1991, sera supportée par la signalisation routière et piếtonnière du Grand Montréal à compter de 1992;

- le Pôle Maisonneuve est désormais reconnu sur le même pied d'égalité que le Centre-ville et que le VieuxMontréal/Vieux-Portparmi les masses critiques touristiques à développer par les divers paliers de gouvernement.

Comme nous l'avons dẹjà dit, l'addition du Biodôme, à compter de 1992, devrait porter l'achalandage du Parc olympique à 4,4 millions d'entrées payantes par année et celui du Pôle Maisonneuve à 5,7 millions. 
Une telle fréquentation à laquelles'ajoutera celle de futurs équipements qu'il reste à déterminer au niveau de la planification stratégique de la Régie de concert avec le ministère du Tourisme, et avec le Jardin botanique en collaboration avec la Ville de Montréal, devrait à moyen terme justifier 1'implantation de facilités de séjour sur le site, compte tenu que la durée de visite de toutes les attractions du Pôle pourra prendre deux ou trois jours.

A cette réalité potentielle s'ajoute le séjour actuel de centaines d'exposants pendant les grands salons d'hiver et la possibilité que ce type de clientèle puisse s'élargir du printemps à l'automne, pendant la saison de baseball, si on utilisait les vastes espaces du Parc olympique pour y implanter les structures d'accueil d'expositions manufacturières dont Montréal a besoin.

Il serait en effet logique de concentrer dans un même environnement de telles facilités commerciales requises par le tourisme d'affaires avec les attractions familiales, puisque cette combinaison motive le choix de villes de destination par une clientèle croissante qui désire allier plaisir et affaires.

Orlando et Las Vegas sont de bons exemples du succes de cette combinaison recherchée par les entreprises et les associations pour optimiser les relations avec leur personnel, ou le nombre d'inscription de leurs membres, et courus par ces derniers parce que les familles peuvent profiter de leurs affaires pour se distraire.

\section{Notre quatrième défi: consolider la vision d'ensemble}

Comme la combinaison plaisir et affaires dans un même lieu favorise le choix de certaines villes de destination par rapport à d'autres, et conme le Pôle Maisonneuve peut offrir cette opportunité à Montréal dans son positionnement face au développement de ce créneau en croissance dans l'industrie touristique, il importe de communiquer ces opportunités aux décideurs de tous les paliers d'intervention pour qu'ils en tiennent compte dans leurs propres plans de développement.

Cette transparence dont fait preuve la direction de la Régie des installations olympiques suppose aussi une certaine restructuration du mode de pensée traditionnel qui a présidé au développement de Montréal depuis un quart de siècle. Il faut privilégier une gestion par objectifs communs en priorité sur les intérêts politiques ponctuels. Si on souhaite vraiment que la notion des «pôles» prenne toute sa force en terme de masses critiques touristiques, il faut leur donner à chacun une mission exclusive. Ainsi, par exemple:

- la mission exclusive du pôle Centreville pourrait être lamise en valeur des attraits culturels, depuis les arts jusqu'au shopping, sans oublier la vie nocturne;

- la mission exclusive du pôle VieuxMontréal/Vieux-Port pourrait être la mise en valeur de notre passé, depuis le régime français jusqu'à l'inauguration de la Voie maritime;

- la mission exclusive du Pôle Maisonneuve pourrait être la mise en valeur de notre avenir, du loisir et du sport à l'environnement incluant toute la vulgarisation scientifique.

- la mission exclusive du pôle Parc-desIles pourrait être la mise en valeur de l'excitation en plein-air, du parc d'amusement aux pique-niques.

- la mission exclusive du pốle MontRoyal/Oratoire pourrait être la mise en valeur de la réflexion, de la flânerie au recueillement.

L'exclusivité de ces missions devrait privilégier des équipements homogènes au sein de chaque pôle et complémentaires entre les divers pôles.

La synergie qui sert de fil conducteur au présent développement du Parc olympique et du Jardin botanique est bien sentie dans ces exemples de vocations cexclusives puisque ces vocations se renforcent mutuellement au profit de la destination Montréal.

Une autre variable qu'il faut privilégier est l'implantation d'attractions accessibles toute l'année. Nos sociétés n'ont plus les moyens financiers d'assumer des sites qui engendrent des dépenses sur douze mois contre des revenus pendant trois ou quatre mois! Cette préoccupation répond au désir des hôteliers du Grand Montréal qui ont grand besoin d'optimiser leur taux d'occupation au pire de la saison basse.

L'adoption de ces principes faciliterait ensuite le développement et la promotion de forfaits (du laissez-passer de deux jours au passeportannuel) pour l'ensemble des pôles touristiques montréalais, comme nous avons l'intention de le faire dans un proche avenir au Parc olympique et au Jardin botanique. Voilà ma perception de notre vision.

Rappelons que notre vision d'ensemble depuis $1986 \mathrm{n}^{\prime} \mathrm{a}$ jamais cessé de prendre en considération notre voisin immédiat quiest en voie de devenir l"un des plus beaux jardins botaniques du monde!

Les groupes d'étudiants constituent 12 à 15 pour cent de son achalandage annuel tout en représentant sa principale clientele hivernale. Le tourisme familial domine du printemps à l'automne, la moitié en provenance de la région et l'autre moitié de l'extérieur. L'ajout du Biodône devrait augmenter substantiellement la part de

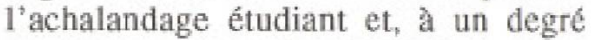
moindre, celle des aînés.

Comme le même profil caractérise les visiteurs du Parc olympique, nous opérons conjointement une navette qui relie gratuitement les deux sites pendant l'été, depuis 1987. Ce service sera éventuellement implanté à l'année longue et il pourrait peut-être évoluer au point de justifier une forme de monorail un jour... Qui sait jusqu'où notre rêve pourra nous conduire?

Le site qu'occupe le Parc olympique est woué aux loisirs et aux sports depuis 1912 , alors que la ville de Maisonneuve, annexée a Montréal depuis, créait un vaste parc récréatif d'une superficie de 204 hectares. On disait alors que ce serait $\alpha_{. .}$selon les plans préparés, un endroit d'une beauté à nulle autre pareille».

La prophétie d'il y a 80 ans s'est réalisée par étapes, avec le Jardin dans les années '30 et avec les Jeux dans les années '70. La vitalité du nouveau partenariat Parc-Jardin fait maintenant du Pôle Maisonneuve la principale masse critique touristique du Quebec.

Ensemble, le gouvernement du Québec et la Ville de Montréal partagent la vision d'un complexe unique au monde dont profiteront des millions de personnes à chaque année, au premier rang desquelles les Montréalais et les Québécois.

Ce sont eux qui animent notre volonte dans certaines remises en question! Mais la pérennité de l'esprit des Jeux de 1976 sera assurée, puisque les monuments laissés en héritage seront utiles aux futures générations. $f$ 